\title{
Jugendliche Diabetiker: Wer viel misst, hat einen besseren $\mathrm{HbA}_{1 c}$
}

\begin{abstract}
Die Blutzuckerselbstmessung (BZSM) ist ein wichtiger Baustein in der effektiven Behandlung von Kindern mit Diabetes. Es gibt aber nur wenige Studien zum Zusammenhang von Messfrequenz und Stoffwechselkontrolle sowie Komplikationen. Auch der Einfluss der Therapieform auf diesen Zusammenhang ist in diesem Alter nicht bekannt. Da bietet es sich an, eine große Datenbank im Hinblick auf diese Fragen zu analysieren.
\end{abstract}

- Die Daten der DPV-Datenbank aus den Jahren 1995-2006 liegen der Analyse zugrunde. In dieser Zeit wurden 26723 Kinder und Jugendliche in 233 deutschen und österreichischen Zentren betreut. Für die Studie wurden für jeden Patienten Durchschnittswerte aus dem aktuellsten Therapiejahr verwendet. AuBer der Zahl der BZSM wurden Geschlecht, Alter, Diabetesdauer, Ketoazidose mit stationärer Aufnahme, Hypoglykämien und $\mathrm{HbA}_{1 \mathrm{c}}$ (mathematisch standardisiert) erfasst. Es erfolgte eine Subanalyse von drei Altersgruppen (0-5 Jahre $=7,4 \%, 6-12$ Jahre $=28,3 \%, 13-18$ Jahre $=64,2 \%)$. Das mittlere Alter lag zuletzt bei 12,7 Jahren, die mittlere Diabetesdauer bei 4,8 Jahren.

Der mittlere $\mathrm{HbA}_{1 \mathrm{c}}$ lag insgesamt bei $8,16 \%$ und war vom Alter $(0-5$ Jahre $=$ $7,59 \%, 6-12$ Jahre $=7,61 \%, 13-18$ Jahre $=8,46 \%)$ und der Therapieform abhän$\operatorname{gig}(\mathrm{CT}=7,94 \%$, $\mathrm{ICT}=8,24 \%$, CSII $=$ $8,01 \%)$. Im Mittel wurde von den Patienten oder deren Eltern der Blutzucker 4,7mal pro Tag gemessen. Von 1995 bis 2006 stieg diese Zahl kontinuierlich von 3,2 auf 5,1 BZSM/d. Dabei wurde am meisten im Kleinkindesalter gemessen $(6 / d)$ und am wenigsten von den Jugendlichen (4,4/d). Pumpenpatienten kontrollierten ihre Werte signifikant häufiger (5,3/d vs. 4,7/d bei ICT). Mehr Messungen waren signifikant mit einer besseren Einstellung assoziiert. Im Schnitt kann mit einer Messung mehr am Tag eine Verbesserung des $\mathrm{HbA}_{1 \mathrm{c}}$ um $0,2 \%$ erreicht werden, mit Pumpe sogar um $0,27 \%$. Ab einer Messungshäufigkeit von fünf Werten am Tag zeigte sich aber keine signifikante Verbesserung mehr. Es gab auch eine signifikante Beziehung zwischen der Häufigkeit von Hypoglykämien und der BZ-Messfrequenz. Die Rate der Hypoglykämien stieg mit der Messfrequenz, für die Zahl von Ketoazidosen war die Beziehung invers.

\footnotetext{
- R. Ziegler et al.

Frequency of SMBG correlates with $\mathrm{HbA}_{1 c}$ and acute complications in children and adolescents with type 1 diabetes. Pediatric Diabetes 12 (2011) 11-17
}

\section{Kommentar}

Die Interpretation dieser Daten muss sehr vorsichtig erfolgen. Es zeigt sich aber eine klare Abhängigkeit der Zahl von BZSM mit der Stoffwechseleinstellung, gemessen am $\mathrm{Hb} \mathrm{A}_{1 \mathrm{c}}$ im Bereich von 0-5 Messungen pro Tag, bei der jede zusätzliche Messung den $H b A_{1 c}$ verbessert. Jugendliche scheinen von der Erhöhung ihrer Messfrequenz mehr zu profitieren, auch über fünf Messungen hinaus. Gerade in diesem Alter ist die Zahl der Messungen ein guter Parameter für die gute Selbstbehandlung des Diabetes, da sie ja eine Dosisanpassung nach der Messung erlaubt. Bei Kleinkindern machen die Eltern meist eine Therapie mit häufigen Messungen (4-7), sodass hier keine so gravierenden Unterschiede in Erscheinung treten. Gerade Pumpenpatienten profitieren aber auch von mehr Messungen, da eine zeitnahe Reaktion auf das Messergebnis ohne wesentlichen zusätzlichen Aufwand (Pen nehmen und spritzen) erfolgen kann.

Bei Patienten, die deutlich mehr messen, zeigt die Messfrequenz möglicherweise die momentan schwierige Stoffwechselsituation mit schwankenden Werten an. Auch die höhere Messfrequenz bei Hypoglykämien ist sicher nicht als kausaler Zusammenhang zu deuten. Bei bekannter Unterzuckerungsneigung wird man sicher öfter den Blutzucker kontrollieren, um ein erneutes Auftreten eines solchen Ereignisses zu vermeiden. Limitiert wird die Studienaussage sicher dadurch, dass es sich um keine randomisierte Studie handelt. Dafür spiegeln die Daten aber das "Real Life" wider.

T. KAPELLEN - 